J. Clin. Chem. Clin. Biochem.

Vol. 16, 1978, pp. 513-517

\title{
Uroporphyrinogen-Decarboxylase in Erythrocyten \\ Untersuchungen zum primären genetischen Enzymdefekt bei chronischer hepatischer Porphyrie
}

Von $R$. v. Tiepermann und M. Doss

Fach Klinische Biochemie im Fachbereich Humanmedizin der Philipps-Universität, Marburg an der Lahn

(Eingegangen am 16. Mai 1978)

Herrn Professor Dr. Dr. Ernst Schütte zum 70. Geburtstag gewidmet

Zusammenfassung: Bei chronischer hepatischer Porphyrie einschließlich deren klinischer Phase, der Porphyria cutanea tarda, ist die Aktivität der Uroporphyrinogen-Decarboxylase nicht nur in der Leber, sondern auch in den Erythrocyten erniedrigt. Die gleichsinnige Aktivitätsminderung dieses Enzyms in Leber und Erythrocyten sowohl bei familiär als auch bei sporadisch vorkommender chronischer hepatischer Porphyrie zeigt an, daß die Uroporphyrinogen-Decarboxylasestörung der primäre genetische Defekt bei chronischer hepatischer Porphyrie ist, welcher wahrscheinlich autosomal dominant vererbt wird. Aber erst hinzukommende Faktoren wie Leberschaden, Alkohol, Östrogene und Neoplasien führen zur klinischen Manifestation der Porphyrinstoffwechselstörung. Der Enzymdefekt bleibt ohne diese zusätzlichen pathogenen Einflüsse kompensiert und hat weder in der Leber noch im Knochenmark eine Störung der Häm- bzw. Hämoglobinsynthese zur Folge.

Uroporphyrinogen decarboxylase in erythrocytes:

Studies on the primary genetic enzyme defect in chronic hepatic porphyria

Summary: In chronic hepatic porphyria, including the clinical phase, porphyria cutanea tarda, the activity of uroporphyrinogen decarboxylase is decreased not only in the liver, but also in the erythrocytes. The synonomous decrease in the enzymic activity in liver and erythrocytes in both familial and sporadic hepatic porphyria shows that the disturbance of this enzyme is the primary genetic defect of this condition; inheritance of the defect is probably autosomal and dominant. The clinical manifestation of disturbances of porphyrin metabolism are precipitated, however, by additional factors, such as liver damage, alcohol, oestrogens and neoplastic growths. In the absence of these other pathogenic influences, the enzyme defect is compensated and does not result in disturbances of haem or haemoglobin synthesis, either in the liver or the bone marrow.

\section{Einfuihrung}

Die charakteristische Akkumulation der Porphyrine im Lebergewebe und das gleichermaßen charakteristische Befundprofil der im Urin ausgeschiedenen Porphyrine bei den chronischen hepatischen Porphyrien wiesen darauf hin, daß der primäre Defekt dieser Stoffwechselerkrankung die hepatische Uroporphyrinogen-Decarboxylase (EC 4.1.1.37) betrifft $(1,2)$. Da weniger als $10 \%$ der Patienten mit chronischen Leberschäden, insbesondere mit chronischer Hepatitis und Lebercirrhose, eine chronische hepatische Porphyrie einschließlich deren Manifestationsform, der Porphyria cutanea tarda, entwickeln, wurde eine genetische Disposition für die biochemische und klinische Pathogenese der chronischen hepatischen Porphyrie vermutet (3). Untersuchungen der Decarboxylierung des Uroporphyrinogens im Leberhomogenat von perkutan gewonnenen Leberbiopsien ergaben, daß in latenten und klinischen Phasen der chronischen hepatischen Porphyrien (4) die Aktivität der UroporphyrinogenDecarboxylase vermindert ist (5). Auf diesen als die primäre enzymatische Störung betrachteten Defekt bei chronischen hepatischen Porphyrien ist die Akkumulation von Uro- und Heptacarboxyporphyrin in der Leber zurückzuführen (1-5); es sind speziell diese Porphyrine, die bei den chronischen hepatischen Porphyrien in groBer Menge im Urin ausgeschieden werden und anhand ihrer charakteristischen Konstellation die Diagnostik der chronischen hepatischen Porphyrinopathien erlauben (1-4).

Wenn der chronischen hepatischen Porphyrie eine hereditäre Transmission zugrundeliegt, dann müßte der primäre Enzymdefekt nicht nur in der Leber, sondern auch - wie bei der akuten intermittierenden Porphyrie in anderen Zellen nachweisbar sein. Unabhängig vonein- 
ander haben Kushner et al. (6) und unsere Arbeitsgruppe den Uroporphyrinogen-Decarboxylase-Defekt auch in den Erythrocyten nachweisen können (7). Der Befund einer verminderten Uroporphyrinogen-DecarboxylaseAktivität in den Erythrocyten bei Porphyria cutanea tarda-Patienten blieb nicht unwidersprochen (8). Sehr wahrscheinlich sind diese Divergenzen methodisch bedingt. Wir konnten sowohl in Biosynthesestudien der gesamten Porphyrinogen- und Hämsynthesekette, die bis zum Koproporphyrin beide Isomerenreihen integrierte (9), als auch mit einer neu entwickelten Enzymbestimmung, die im folgenden beschrieben wird, eine Verminderung der Uroporphyrinogen-DecarboxylaseAktivität sowohl bei sogenannten, sporadischen' Porphyria cutanea tarda-Patienten als auch bei familiärem Vorkommen von chronischer hepatischer Porphyrie einschließlich der Porphyria cutanea tarda feststellen $(10,11)$.

\section{Untersuchungsgut und Methodik}

Zur Untersuchung der Uroporphyrinogen-Decarboxylase-Aktivität wurden die Erythrocyten aus heparinisierten Blutproben verwendet. Die Probenvorbereitung erfolgte analog derjenigen zur Bestimmung der Uroporphyrinogen-Synthase-Aktivität in Erythrocyten (12). Die Erythrocyten wurden durch dreimaliges wechselweises Einfrieren bei minus $30^{\circ} \mathrm{C}$ und Auftauen (bei Raumtemperatur) hämolysiert.

Prinzip der Uroporphyrinogen-DecarboxylaseBestimmung

Das methodische Prinzip geht auf die Untersuchungen der Uroporphyrinogen-Decarboxylase (in der Mäusemilz) von Romeo \& Levin zurück (13). Es wird die Decarboxylierung von Uroporphyrinogen I zu Koproporphyrinogen gemessen. Uroporphyrinogen I wird im Hämolysat (also endogen) aus zugesetztem Phorphobilinogen synthetisiert. Demzufolge sind zwei Inkubationen des Erythrocyten-Hämolysats erforderlich:

1. Synthese des Substrates Uroporphyrinogen I und

2. Decarboxylierung von Uroporphyrinogen I zu Koproporphyrinogen I, das in seiner oxidierten Form nach dünnschichtchromatographischer Isolierung spektrophotometrisch gemessen wird.

Durchführung der Bestimmung

Synthese von Uroporphyrinogen I

Das im Verhältnis 1:5 mit Phosphatpuffer (133 mmol/1, pH 7,65) verdünnte Erythrocyten-Hämolysat wurde bei $65^{\circ} \mathrm{C} 15$ Minuten erhitzt (Inaktivierung der Uroporphyrinogen-Cosynthase und -Decarboxylase). Nach Abkühlen im Eisbad wurde Glutathion in einer Endkonzentration von $2 \mathrm{mmol} / \mathrm{l}$ und Porpho-

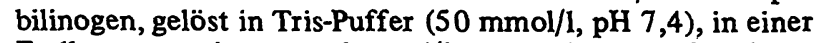
Endkonzentration von $50 \mu \mathrm{mol} / 1$ hinzugefügt. Die Synthese von Uroporphyrinogen I erfolgte bei $45^{\circ} \mathrm{C}$ über 60 min unter Ausschluß von Licht.

\section{Messung der Uroporphyrinogen-Decarboxylase-Aktivität}

Der Uroporphyrinogen I enthaltenden Probe wurde mit $4 \mathrm{Vol}$. $140 \mathrm{mmol} / 1 \mathrm{KH}_{2} \mathrm{PO}_{4}$-Lösung verdünntes Ery throcy ten-Hämolysat hinzugesetzt. Damit wurde der $\mathrm{pH}$ auf 6,8 erniedrigt (13). Der Ansatz wurde bei $37^{\circ} \mathrm{C} 60 \mathrm{~min}$ inkubiert, danach sofort bei $-40^{\circ} \mathrm{C}$ eingefroren und lyophilisiert.

Das synthetisierte Koproporphyrin I wurde nach den in dieser Zeitschrift bereits beschriebenen Methoden bestimmt (14).

Als Maß für die Aktivität der Uroporphyrinogen-Decarboxylase wurde definiert: $\mathrm{U}=\mu \mathrm{mol} / \mathrm{h} \cdot 1$ Erythrocyten gebildetes Koproporphyrin.

\section{Ergebnisse und Diskussion}

\section{Methodische Aspekte}

Der kritische Schritt zur Uroporphyrinogen-Decarboxylase-Aktivitätsbestimmung betrifft die Herstellung des Substrats. Das Substrat Uroporphyrinogen wird aus exogenem Porphobilinogen im Erythrocy tenhämolysat synthetisiert. Somit resultiert die Bestimmung der Uroporphyrinogen-Decarboxylase aus zwei Reaktionen: Synthese des Substrates für die UroporphyrinogenDecarboxylase und Synthese des Produktes der Uroporphyrinogen-Decarboxylase.

Sowohl Romeo \& Levin (13) als auch Kushner et al. (6) konnten zeigen, daß die Decarboxylierung des Uroporphyrinogens in den beiden Isomerenreihen I und III sowohl in den Erythrocyten vom Menschen als auch in der Milz von Mäusen gleichsinnig verläuft. Insofern besteht zwischen der enzymatischen Decarboxylierung von Uroporphyrinogen I und III kein Unterschied. Wenn man in einem methodischen Ansat $z$ von aus exogenem Porphobilinogen endogen generiertem Uroporphyrinogen ausgeht, muß man in der Synthesesequenz der Isomerenreihe I arbeiten, um Interferenzen der Folgeenzyme der UroporphyrinogenSynthase im eigentlichen Enzymschritt, nämlich der Bestimmung der Uroporphyrinogen-Decarboxylase, auszuschließen. Zudem hat die Verwendung von Uroporphyrinogen I den Vorteil, daß dieser Synthesezweig auf der Stufe des Koproporphyrinogen I bereits physiologischerweise endet, so daß a priori eine weitere enzymatische Utilisation des synthetisierten Koproporphyrinogens ausgeschlossen ist. Der Vorteil der hier beschriebenen Methode geht zum ersten Mal von einer Uroporphyrinogensynthese im Erythrocy tenhämolysat des Menschen aus, während die früher beschriebenen Methoden Uroporphyrinogen I mit Hilfe einer aus Mäusemilz isolierten Uroporphyrinogen-Synthase herstellten. Das vorliegende Verfahren macht sich die sog. ,Hitzestabilität' der Uroporphyrinogen-Synthase zunutze und erlaubt somit eine Doppelinkubation mit Erythrocytenhämolysat derselben Herkunft ohne vorausgegangene Enzymisolierung.

Kinetische Studien zum Einfluß der Vorinkubation auf die Hauptinkubation ergaben, daß eine maximale Synthese des Gesamtkoproporphyrins (Isomer I und III) nach ca. 30 min erfolgte (Abb. 1a), wobei der relative Anteil des Koproporphyrins I innerhalb des Gesamtkoproporphyrins mit der Inkubationsdauer zunimmt: sein Anteil erreicht nach einer Stunde der Vorinkubation 90\%.(Abb. 1b). Der Restanteil von Koproporphyrin-İsomer III ist auf eine Synthese von Koproporphyrinogen III in der Hauptinkubation mit in der Vorinkubation nicht verbrauchtem Porphobilinogen zurückzuführen. Das in der Vorinkubation zur Synthese von Uroporphyrinogen I zugesetzte Porphobilinogen wird zu über $90 \%$ in 60 Minuten verbraucht (Abb. 2). Der Rest wird zwar von dem in der Hauptinkubation 

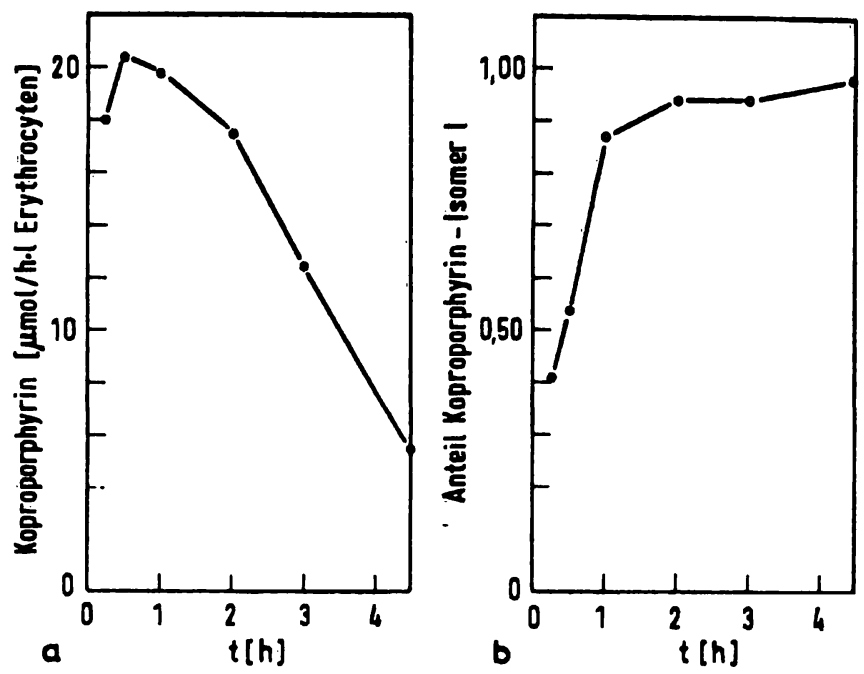

Abb. 1. Kinetische Untersuchungen zur UroporphyrinogenDecarboxylase-Bestimmung in Ery throcy ten.

a) Abhängigkeit der Aktivität der UroporphyrinogenDecarboxylase $(\mu \mathrm{mol} / \mathrm{h} \cdot 1$ Ery throcy ten gebildetes Koproporphyrin) von der Dauer der Vorinkubation ( 1 bis 4 h): Ermittlung der Bedingungen zur optimalen Substratsynthese.

b) I somerenverteilung des zum Koproporphyrinogen decarboxylierten Uroporphyrinogen.

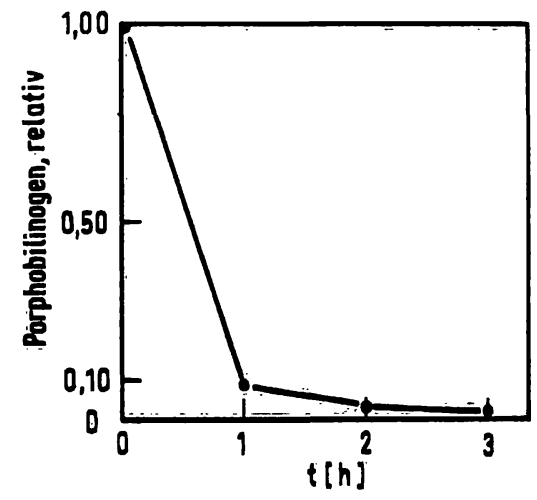

Abb. 2. Porphobilinogen-Verbrauch während der Vorinkubation zur Uroporphyrinogensynthese.

mit neuem Erythrocytenhämolysat intakten Enzymsyștem zu Porphyrinogenen einșchließlich des Koproporphyrinogens III um̈gesetżt (Abb. 1b), stört jedoch die Aussage des Enzym-Assays nicht, wenn man den Inkubationszeitraum von einer Stunde in der Hauptinkubation nicht überschreitet. Kinetische Untersuchungen der Sỳnthese vón Uróporphyrinogen I in der Vorinkubation zeigen einen nahezu linearen Anstieg der Syntheșerate von Uroporphyrinogen I innerhalb der ersten Stunde (Abb. 3).

Dem Ansatz zur Vorinkubation wird reduziertes Glutathion zugesetżt, um dàs frisch synthetisierte und leicht oxidable Uroporphyrinogen I vor irreversibler Oxidation zu schützen und für die UroporphyrinogenDecarboxylase-Reaktion in der Hauptinkubation verfügbar zu halten. Die Konzentration des synthetisierten

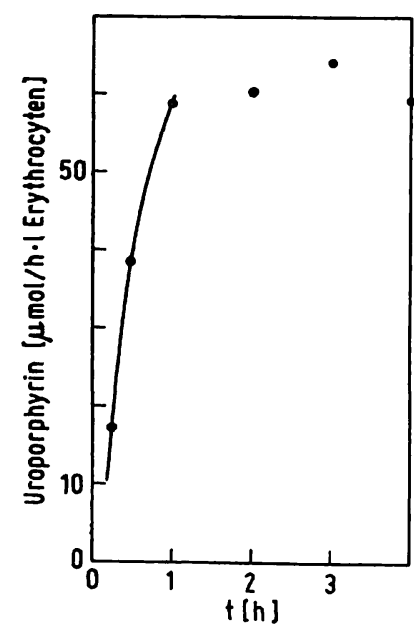

Abb. 3. Synthese von Uroporphyrinogen (Substrat der Uroporphyrinogen-Decarboxylase), bestimmt als Uroporphyrin spek trophotometrisch (erster Inkubationsschritt).

Koproporphyrinogens zeigt unter den für die Hauptinkubation gewählten Bedingungen einen linearen Verlauf (Abb. 4). Zwar steigt die Synthese bis zu zwei Stunden weiterhin an, stagniert jedoch danach, da das aus der Vorinkubation gewonnene Substrat verbraucht ist. Die Substratsättigung des Enzyms ist nach einer Stunde unter den gewählten Voraussetzungen nicht mehr gegeben.

Die Präzision der Uroporphyrinogen-DecarboxylaseBestimmung liegt in der Serie bei 4\% und in der Analytik von Tag zu Tag bei $9 \%(n=10)$. Das Enzym ist bis zu etwa drei Tagen bei Raumtemperatur stabil, so daß eine Versendung der Blutproben in ein Speziallaboratorium möglich ist. Die dann bei ca. minus $25^{\circ} \mathrm{C}$ eingefrorenen Erythrocyten-Proben weisen eine gleichbleibende Enzymaktivität über mindestens einen Monat auf.

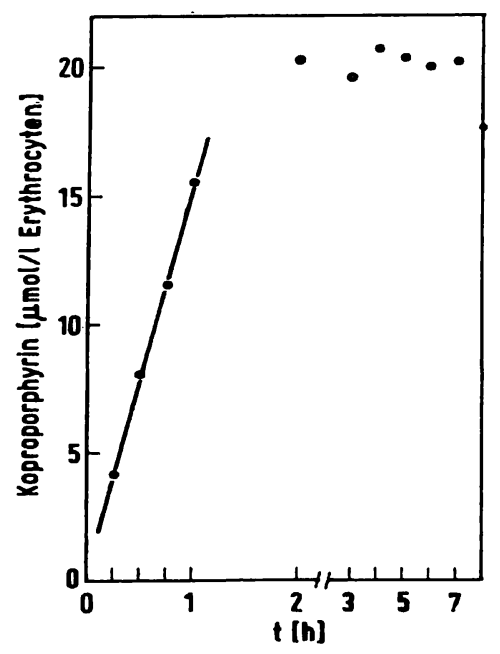

Abb. 4. Synthese von Koproporphyrinogen (Produkt der Uroporphyrinogen-Decarboxylase-Reaktion: zweiter Inkubationsschritt), bestimmt als Koproporphyrin spektrophotometrisch. 


\section{Diagnostische Aspekte}

Bereits in den früheren Arbeiten unseres Arbeitskreises über die biochemische Pathogenese der chronischen hepatischen Porphyrie wurde anhand sowohl von Metabolitstudien in Leber und Urin als auch von Biosynthesestudien im Leberbiopsiegewebe postuliert, daß der chronischen hepatischen Porphyrie ein Uroporphyrinogen-Decarboxylase-Defekt zugrunde liegen muß, der jedoch erst im Verlaufe des Lebens manifest wird, wenn zusätzliche exogene Faktoren die Störung aus der genetischen in die latente und letztlich in die klinische Phase transformieren (1-5). Diese Faktoren sind Leberschaden, Alkohol und Östrogene $(1,3,15)$. Untersuchungen an Leberhomogenaten von Patienten mit chronischer hepatischer Porphyrie (5) und sowohl die früheren $(7,9)$ als auch die jetzigen Untersuchungen zur Uroporphyrinogen-Decarboxylase in Erythrocyten zeigen eindeutig, daß die Aktivitätsminderung des Enzyms als primärer Defekt bei den chronischen hepatischen Porphyrien betrachtet werden muß. Familienuntersuchungen lassen den Schluß zu, daß der Defekt wahrscheinlich in einer autosomal dominanten Weise vererbt wird $(6,16)$. Desweiteren lassen Familienuntersuchungen, welche zum Teil drei und vier Generationen umfassen, erkennen, daß der Defekt nicht nur bei Erwachsenen, sondern auch bei Kindern gefunden werden kann, ohne daß wesentliche Normabweichungen der Porphyrinausscheidung simultan schon bestehen. Wahrscheinlich darf davon ausgegangen werden, daß die meisten der bisher diagnostizierten, sporadischen' Porphyria cutanea tarda-Fälle auch genetisch prädisponiert sind. Das in den vergangenen Jahren häufigere Auftreten von Porphyria cutanea tarda bei jungen Frauen, die orale Kontrazeptiva einnehmen, unterstützt die Auffassung, daß der genetisch angelegte Enzymdefekt der Uroporphyrinogen-Decarboxylase erst durch exogene Faktoren klinisch wirksam wird (15). Die Porphyria cutanea tarda galt lange Zeit als eine überwiegend bei Männern im Alter von über $40 \mathrm{Jahren}$ vorkommende Erkrankung; neuere Beobachtungen widersprechen dieser Auffassung.

Die Aktivität der Uroporphyrinogen-Decarboxylase ist gegenüber einer Kontrollgruppe signifikant erniedrigt (Tab. 1): Im Mittel liegt die Aktivität der Uroporphyrinogen-Decarboxylase in den Erythrocyten von Patienten mit chronischer hepatischer Porphyrie bei 57\% im Vergleich zum Normalkollektiv. Bei fünf Patienten mit Porphyria cutanea tarda lag die Aktivität der erythrocytären Uroporphyrinogen-Decarboxylase im Normalbereich. Analoge Befunde wurden im Hinblick auf die Aktivität der Uroporphyrinogen-Synthase auch bei akuter intermittierender Porphyrie erhoben (12). Für diese Befunde haben wir noch keine Erklärung. Generell darf davon ausgegangen werden, daß der Enzymdefekt in den Erythrocyten sowohl bei akuter intermittierender Porphyrie als auch bei chronischer hepatischer Porphyrie keine Störung der Hämsynthese zur Folge hat, lediglich einen ,genetischen Marker' darstellt und
Tab. 1. Aktivität der Uroporphyrinogen-Decarboxylase in Erythrocy ten von Patienten mit chronischer hepatischer Porphyrie (CHP) und Personen ohne Porphyrinstoffwechselstörung.

\begin{tabular}{|c|c|c|c|}
\hline \multirow[b]{2}{*}{$\begin{array}{l}\text { CHP-Familien } \\
\text { CHP-Patienten, sporadisch" }\end{array}$} & \multirow[b]{2}{*}{$\begin{array}{l}(n=27) \\
(n=32)\end{array}$} & \multicolumn{2}{|c|}{ 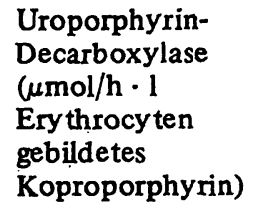 } \\
\hline & & $\begin{array}{l}11,9 \pm 3,5 \\
10,7 \pm 3,1\end{array}$ & $\begin{array}{l}(\bar{x} \pm s) \\
(\bar{x} \pm s)\end{array}$ \\
\hline $\begin{array}{l}\text { Summe CHP } \\
\text { Kontrollen }\end{array}$ & $\begin{array}{l}(n=59) \\
(n=120)\end{array}$ & $\begin{array}{l}11,2 \pm 3,3 \\
19,6 \pm 3,1\end{array}$ & $\begin{array}{l}(\bar{x} \pm s) \\
(\bar{x} \pm s)\end{array}$ \\
\hline $\begin{array}{l}\bar{x}_{\text {CHPp }} \sqrt{\mathbf{x}}_{\text {Kontrollen }} \\
\text { Signifikanz }\end{array}$ & $\begin{aligned} & =0,57 \\
p & <0,001\end{aligned}$ & & \\
\hline
\end{tabular}

die für die Porphyrie ausschlaggebende Stoffwechselsituation der Porphyrinogen- und Hämșynthese in der Leber nicht reflektiert. Die Frage, ob rein exogen induzierte chronische hepatische Porphyrien, z. B. durch Hexachlorbenzol oder andere polychlorierte oder polybromierte aromatische Kohlenwasserstoffe, auch eine Störung der Uroporphyrinogen-Decarboxylase in den Erythrocyten auslösen können - in der Leber ist dieser toxische Defekt mehrfach experimentell belegt worden $(17,18)=$, ist gegenwärtig eine noch offene Frage. Ein Uroporphyrinogen-Decarboxylase-Defekt nach Hexachlorbenzol-Intoxikation der Ratte konnte in der Milz bislang nicht eindeutig bewiesen werden (19). Vorläufige experimentelle Untersuchungen an alkoholbelasteten Ratten schließen eine Wirkung des Alkohols auch auf die ery throcy täre UroporphyrinogenDecarboxylase nicht aus (20). Die potenzierende Wirkung von Alkohol bei der biochemischen und klinischen Manifestation der chronischen hepatischen Porphyrie des Menschen ist durch eine Vielzahl klinisch-biochemischer Untersuchungen verschiedener Arbeitsgruppen bewiesen (21): chronischer Alkoholkonsum sowie Alkoholexzess führen zu einer unmittelbaren Steigerung der Uround Heptacarboxyporphyrinausscheidung im Urin, während Alkoholkarenz eine der wesentlichsten Voraussetzungen zur Therapie und Prävention der Porphyria cutanea tarda ist. Insofern muß für den Alkoholeffekt bei chronischer hepatischer Porphyrie eine Wirkung auf die Uroporphyrinogen-Decarboxylase postuliert werden, wobei im Augenblick noch offen bleibt, ob es sich hierbei ausschließlich um eine Wirkung auf eine genetisch vorgeschädigte Uroporphyrinogen-Decarboxylase handelt oder ob unter Alkoholabusus auch eine genetisch nicht-alterierte UroporphyrinogenDecarboxylase so weit chronisch gestört werden kann, daß sich daraus eine chronische hepatische Porphyrie zu entwickeln vermag (21). Diese Frage bedarf im Żusammenhang mit denjenigen Porphyria cutanea tardaFällen, die keine Verminderung der UroporphyrinogenDecarboxylase-Aktivität in den Ery throcyten aufwiesen, noch einer Klärung.

Die Arbeit wurde mit Unterstützung der Deutschen Forschungsgemeinschaft durchgeführt (Do 134/9). 


\section{Literatur}

1. Doss, M. (1970), Hoppe-Seyler's Z. Physiol. Chem. 351, $1300-1301$

2. Doss, M. (1971), Klin. Wochenschr. 49, 941-942.

3. Doss, M., Look, D., Henning, H., Nawrocki, P., Schmidt, A., Dölle, W., Korb, G. \& Lüders, C. J. (1972), Klin. Wochenschr. 50, 1025-1032.

4. Doss, M., Look, D., Henning, H., Lüders, C. J., Dölle, W. \& Strohmeyer, G. (1971), J. Clin. Chem. Clin. Biochem. 9, 471-477.

5. Doss, M., Schermuly, E., Look, D. \& Henning, H. (1976), in: Porphyrins in Human Diseases (Doss, M. ed.) pp. 286-298, Karger, Basel.

6. Kushner, J. P., Barbuto, A. J. \& Lee, G. R. (1976), J. Clin. Invest. S8, 1089-1097.

7. Doss, M., Tiepermann, R. v., Schermuly, E., Scheffer, B. \& Martini, G. A. (1976), in Porphyrins in Human Diseases Report of the Discussions, Suppl. Proc. 1st Int. Porphyrin Meeting (Doss, M. \& Nawrocki, P., eds.), pp. 222-237. Dr. Falk, Freiburg.

8. Blekkenhorst, G., Pimstone, N. R. \& Eales, L. (1976), in Porphyrins in Human Diseases (Doss, M. ed.), pp. 299-311, Karger, Basel.

9. Schermuly, E. \& Doss, M. (1976), Ann. Clin. Res. 8, Suppl. $17,92-102$.
10. Tiepermann, R. v., Doss, M. \& Leonhardt, K. F. (1978), J. Clin. Chem. Clin. Biochem. 16, 47-47.

11. Tiepermann, R. v., Topi, G. C., D'Alessandro Gandolfo, L. \& Doss, M. (1978), J. Clin. Chem. Clin. Biochem. 16, $52-54$.

12. Doss, M. \& Tiepermann, R. v. (1978), J. Clin. Chem. Clin. Biochem. 16,111-118.

13. Romeo, G. \& Levin, E. Y. (1971), Biochim. Biophys. Acta $230,330-341$.

14. Doss, M. (1970), Z. Klin. Chem. Klin. Biochem. 8, 197207.

15. Doss, M. (1977), Dtsch. Med. Wochenschr. 23, 875-877.

16. Tiepermann, R. v., Doss, M., Leonhardt, K. F., Lehr, P. A., D'Alessandro Gandolfo, L. \& Topi, G. C. (1978), Therapiewoche im Druck.

17. Strik, J. J. T. W. A. (1978), in Diagnosis and Therapy of Porphyrias and Lead Intoxication (Doss, M., ed.), im Druck, Springer, Berlin-Heidelberg-New York.

18. Elder, G. H. (1976), Essays Med. Biochem. 2, 75-114.

19. Doss, M., Schermuly, E. \& Koss, G. (1976), Ann. Clin. Res. 8, Suppl. 17, 171-181.

20. Doss, M., Tiepermann, R. v. \& Tetschke, R. (1978), unveröffentlichte Ergebnisse.

21. Doss, M. (1978), Leber-Magen-Darm, im Druck.

Prof. Dr. M. Doss

Fach Klinische Bicchemie im FB Humanmedizin der Philipps-Universität Pilgrimstein 2

3550 Marburg an der Lahn 
. 